\title{
Measurements of Viscoelastic Properties of Linear Polymers in Dilute and Semidilute Solutions with a Flow Birefringence Method
}

\author{
Fumitoshi SUzUKI, Koichiro HORI, ${ }^{* 1}$ Norio KOZUKA, ${ }^{* 2}$ \\ Hideaki KomOda, ${ }^{* 3}$ Kimiaki Katsuro, ${ }^{* 4}$ Yoshiaki TAKAHASHI, \\ Ichiro NODA, and Mitsuru NAGASAWA \\ Department of Synthetic Chemistry, Nagoya University, \\ Furo-cho, Chikusa-ku, Nagoya 464, Japan
}

(Received July 8, 1986)

\begin{abstract}
To study viscoelastic properties of flexible macromolecules in dilute and semidilute solutions, a birefringence instrument with high sensitivity was constructed. A circularly polarized laser beam is used as an incident light, and the distortion of the beam by polymer molecules in shear flow was detected by a rotating half wave plate and photomultiplier and compensated with a Babinet-Soleil plate. The birefringence $\Delta n$ and extinction angle $\chi$ of polymer solutions can be measured simultaneously and independently. The steady-shear viscosity $\eta$ and steady-state compliance $J_{\mathrm{e}}$ can be evaluated from observed values of $\Delta n$ and $\chi$ using a stress-optical law. The reliability of these data was confirmed by comparing the data with those determined by a Maron-Krieger-Sisko capillary viscometer and a Weissenberg rheogoniometer. The concentration dependence of reduced steady-state compliance $J_{\mathrm{eR}}$ of linear polystyrenes in bromobenzene (a matching solvent) in dilute and semidilute solutions are reported.
\end{abstract}

KEY WORDS Viscoelastic Properties / Polystyrene / Dilute Solution / Semidilute Solution / Flow Birefringence /

The viscoelastic properties of polymer solutions may be expressed in terms of two parameters relating with energy dissipation and storage processes, such as steady-shear viscosity $\eta$ and steady-state compliance $J_{\mathrm{e}}$. The viscosity of polymer solutions can be measured easily over a wide range of polymer concentration using capillary or rotational viscometers. $J_{\mathrm{e}}$ can also be determined without difficulty if the polymer concentration is high. In a steady shear flow, they can be determined by measuring shear stress $P_{12}$ and primary normal stress difference $P_{11}-P_{22}$. That is,

$$
\begin{gathered}
P_{12}=\eta \dot{\gamma} \\
P_{11}-P_{22}=2 J_{\mathrm{e}} \eta^{2} \dot{\gamma}^{2}
\end{gathered}
$$

and $J_{\mathrm{e}}$ is defined by

$$
J_{\mathrm{e}}=\left(P_{11}-P_{22}\right) / 2 P_{12}^{2}
$$

where $\dot{\gamma}$ is the shear rate. [See the notes -attached to eq $4 b$.]

In dilute or semidilute solutions, however, ordinary mechanical methods are not sensitive enough for determination of $J_{\mathrm{e}}$. Only a few experiments have been carried out to study the elastic properties of polymer solutions. In dilute solutions the flow birefrin-

Present address: ${ }^{* 1}$ Mitsubishi Electric Corporation, 4-1, Mizuhara, Itami, Hyogo, 664; ${ }^{* 2}$ Toyota Motor Co., Ltd., 1, Toyota-cho, Toyota, Aichi 471; ${ }^{* 3}$ Matsushita Electric Industries Co., Ltd., 1006, Kadoma, Osaka 571; ${ }^{* 4}$ Aito Kogyo Co., Ltd., 24-5, Kitahama-cho, Chita, Aichi 478. 
gence method ${ }^{1,2}$ may be most promising since the rheo-optical method has a much higher sensitivity than mechanical methods.

In the flow birefringence we can observe the refractive index difference between two principal axes, that is, the birefringence $\Delta n$, and the angle between the principal axis and the direction of shear flow, that is, the extinction angle $\chi$. If the principal axis of optical strain tensor agrees with that of stress tensor, $\Delta n$ and $\chi$ are related to shear stress $P_{12}$ and primary normal stress difference $P_{11}-$ $P_{22}$ by the stress-optical law, as follows, ${ }^{1,2}$

$$
\begin{gathered}
P_{12}-\eta_{s} \dot{\gamma}=\Delta n \cdot \sin 2 \chi / 2 C \\
P_{11}-P_{22}=\Delta n \cdot \cos 2 \chi / C
\end{gathered}
$$

where $C$ is the stress-optical coefficient and $\eta_{\mathrm{s}}$ is the solvent viscosity.

To make the stress-optical law valid in polymer solutions the influence of form anisotropy should be removed by using a matching solvent, in which the refractive index increment $\partial n / \partial c$ is negligibly small. Thus, if a matching solvent can be found and the birefringence of the solvent is negligibly small compared with that of polymer, $\eta$ and $J_{\mathrm{e}}$ of polymer solutions are given by ${ }^{1,2}$

$$
\begin{aligned}
\eta & =P_{12} / \dot{\gamma}=\eta_{\mathrm{s}}+\Delta n \cdot \sin 2 \chi / 2 C \dot{\gamma} \\
J_{\mathrm{e}} & =\left(P_{11}-P_{22}\right) / 2 P_{12}^{2} \\
& =\Delta n \cdot \cos 2 \chi / 2 C \eta^{2} \dot{\gamma}^{2}
\end{aligned}
$$

Strictly speaking, the steady-state compliance $J_{\mathrm{e}}$ is defined as the limiting value at negligible shear rate. The value calculated from eq $4 \mathrm{~b}$, which may be dependent on $\dot{\gamma}$, is called as the apparent steady-state compliance $J_{\mathrm{s}}$ in this paper.

For this purpose we designed and constructed a flow birefringence apparatus with high sensitivity, so that both $\Delta n$ and $\chi$ can be determined simultaneously and independently. The principle of the method and a preliminary model of the apparatus were already reported. ${ }^{3}$ In this paper, we report a modified model of the apparatus and some experimental results obtained by the apparatus for polystyrene solutions.

\section{EXPERIMENTAL}

\section{Materials}

Two samples of polystyrene with narrow molecular weight distributions were purchased from Toyo-Soda Manufacturing Co., Ltd. (F-288 and F-450). Their weight-averaged molecular weights are $2.89 \times 10^{6}$ and $4.48 \times 10^{6}$, respectively, according to the manufacturer. The measurements were carried out in bromobenzene, which is a good and matching solvent for polystyrene. Concentration ranges employed here were $0.6 \sim$ $1.4 \mathrm{~g} \mathrm{dl}^{-1}$ for F-288 and $0.4 \sim 1.3 \mathrm{~g} \mathrm{dl}^{-1}$ for F-450. The critical concentration $c^{*}$ where the polymer coils begin to overlap with each other is defined by

$$
c^{*}=3 M / 4 \pi\left\langle s^{2}\right\rangle^{3 / 2} N_{\mathrm{A}}
$$

where $M$ and $\left\langle s^{2}\right\rangle$ are the molecular weight and the mean square radius of gyration of the polymer, respectively, and $N_{\mathrm{A}}$ is Avogadro's number. Assuming that the $\left\langle s^{2}\right\rangle$ of polystyrene in bromobenzene is equal to that of polystyrene in toluene ${ }^{4}$ since both solvents are good for polystyrene, we have $c^{*}=0.209$ $\mathrm{g} \mathrm{dl}^{-1}$ for F-288 and $0.154 \mathrm{~g} \mathrm{dl}^{-1}$ for F-450. Therefore, the concentration regions employed here are in the semidilute region for thermodynamic properties of polymer solutions ${ }^{5,6}$ but in a transition region from dilute to semidilute region for viscosity. ${ }^{7,8}$

\section{Apparatus and Measurements}

Figure 1 shows a block diagram of the optical alignment of the flow birefringence apparatus designed by Matsuura et al. ${ }^{3}$ In this system the circularly polarized laser beam is employed as the incident light. The light with $633 \mathrm{~nm}$ of wavelength from a $2 \mathrm{~mW} \mathrm{He}-\mathrm{Ne}$ gas laser of NEC Co., Ltd., type GLG5321/ GLS5340, is linearly polarized through a po- 


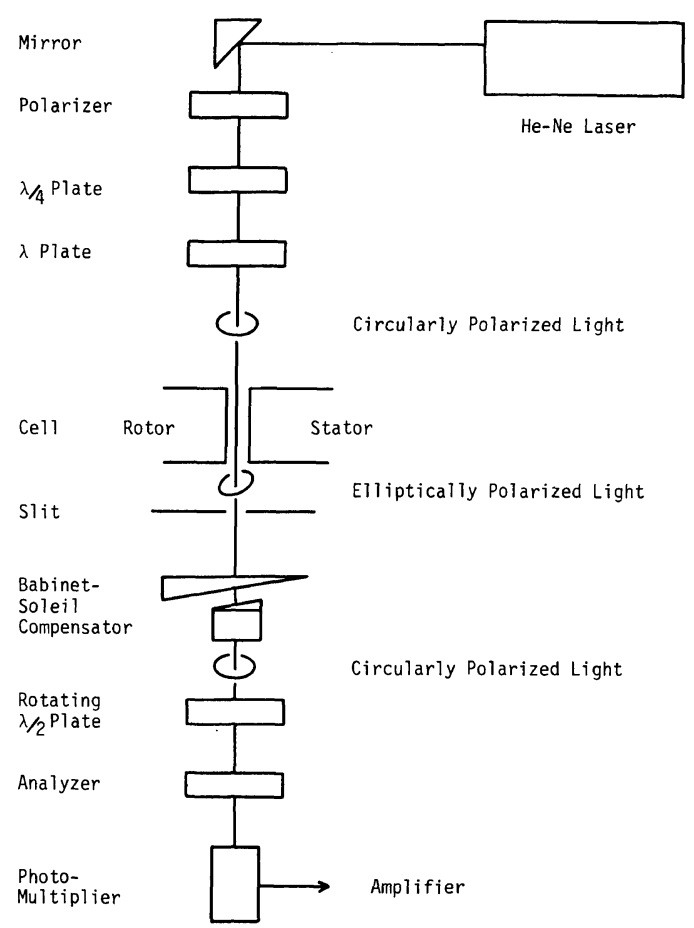

Figure 1. Optical alignment for the flow birefringence apparatus.

larizer. The linearly polarized light is converted to a circularly polarized light by two birefringent plates, a quarter wave plate and a wave plate producing a small retardation angle. The second plate is employed for fine adjustments, since this apparatus demands a highly circularly polarized light with eccentricity less than $2 \times 10^{-4}$. If the polymer solution in the cell has a birefringence, the incident light polarized circularly becomes an elliptically polarized light after transmitting through the cell. The difference between this elliptically polarized and original circularly polarized lights is converted by a rotating half wave plate and a fixed analyzer to a light with oscillatory intensity, which is detected as output of alternating current from a photomultiplier. The difference is then compensated by a micrometer-driven Babinet-Soleil plate (B-S plate) so that the output of photomultiplier become zero. The optical retardation

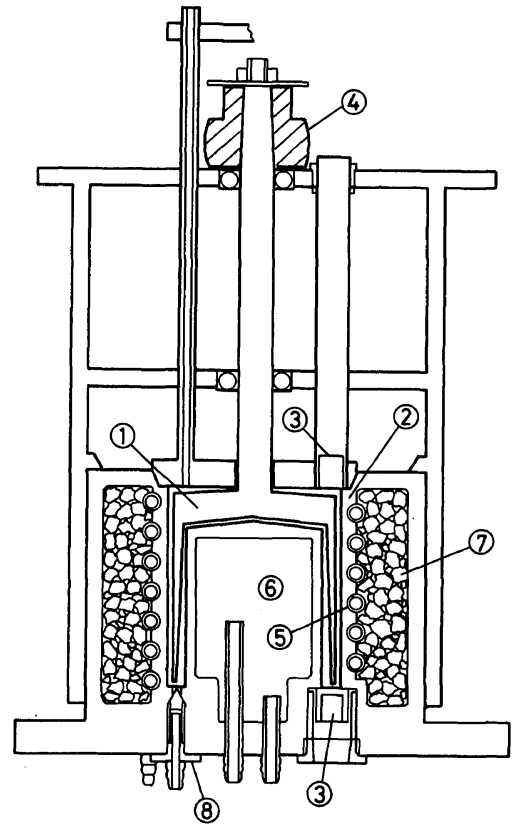

Figure 2. Cross-section of the rotor and the stator in the flow birefringence apparatus. 1, rotor; 2 , stator; 3 , window; 4, pulley; 5, thermostating jacket; 6, thermostating chamber; 7 , thermal insulator; 8 , needle valve.

angle $\delta$ and the extinction angle $\chi$ are obtained from the displacement of micrometer and the rotating angle of B-S plate for compensation, respectively.

The birefringence $\Delta n$ is evaluated from the retardation angle $\delta$ by;

$$
\Delta n=(\lambda / 2 \pi L) \delta
$$

where $\lambda$ is the wavelength and $L$ is the length of optical path in the birefringence medium.

A cross-section of the flow birefringence cell is shown in Figure 2. The cell is an inner rotor type made of stainless steel. The diameter of the rotor is $89.5 \mathrm{~mm}$ and the length is $112 \mathrm{~mm}$. The gap between the rotor and the stator is $0.5 \mathrm{~mm}$. The surface of the rotor was polished as smoothly as possible for stainless steel to eliminate noise due to irregular reflection of the laser beam. The glass windows were fixed by an optical contact method to eliminate optical strains.

The pulley with the diameter of $60 \mathrm{~mm}$ is 
mounted on the upper part of the rotor, and the rotor is belt-driven by a direct current motor regulated by a speed controller of Yasukawa Electric Co., Ltd., UGPMEN16DAB type. The rotating speed can be continuously changed up to $2500 \mathrm{rpm}$, which corresponds to about $4600 \mathrm{~s}^{-1}$ of shear rate. The rotating speeds are measured by counting photo-pulses from 24 holes along circumference on a disk fixed on the pulley. The counting of photo-pulses is carried out with a photointerrupter.

The minimum measurable displacement of the micrometer is $1 \mu \mathrm{m}$, which corresponds to $1 \times 10^{-3}$ radian of the retardation angle of the B-S plate. Introducing $\lambda=633 \mathrm{~nm}, L=112$ $\mathrm{mm}$, and $\delta=1 \times 10^{-3}$ radian into eq 6 , we have $1 \times 10^{-9}$ for the minimum measurable $\Delta n$. However, the sensitivity of $\Delta n$ is one order higher than this measurable limit $\left(\sim 10^{-10}\right)$.

The measurements of flow birefringence were carried out at room temperature $(20$ $\left.25^{\circ} \mathrm{C}\right)$.

\section{RESULTS}

Figure 3 shows an example of the shear rate dependence of $\Delta n$ and $\chi$ determined by this apparatus. The ranges of experimental error are shown as the vertical bars in the figure. Figure 4 shows the shear rate dependence of

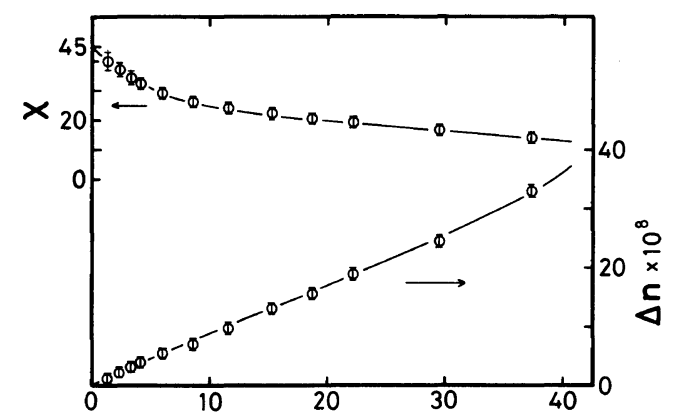

Figure 3. An example of shear rate dependence of birefringence $\Delta n$ and extinction angle $\chi$ of polystyrene in bromobenzene. Sample, F-288; concentration, $0.566 \mathrm{~g} \mathrm{dl}^{-1}$. shear stress $P_{12}$ and primary normal stress difference $P_{11}-P_{22}$ evaluated from the data of $\Delta n$ and $\chi$ by assuming $C=5.75 \times 10^{-10} \mathrm{~cm}^{2}$

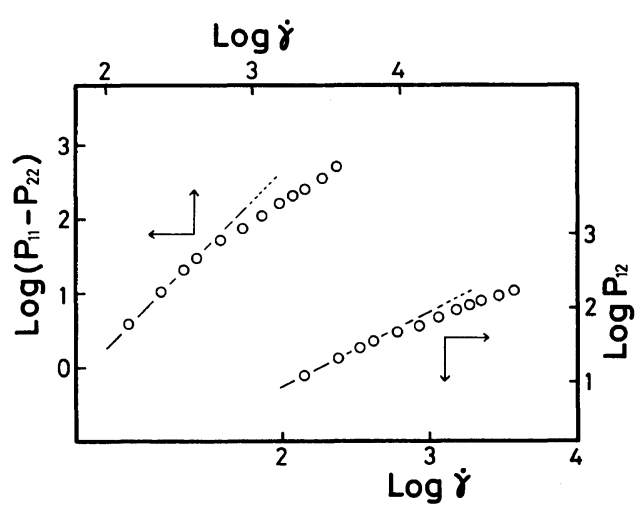

Figure 4. Shear rate dependence of shear stress $P_{12}$ and primary normal stress difference $P_{11}-P_{22}$ calculated from the flow birefringence data in Figure 3.

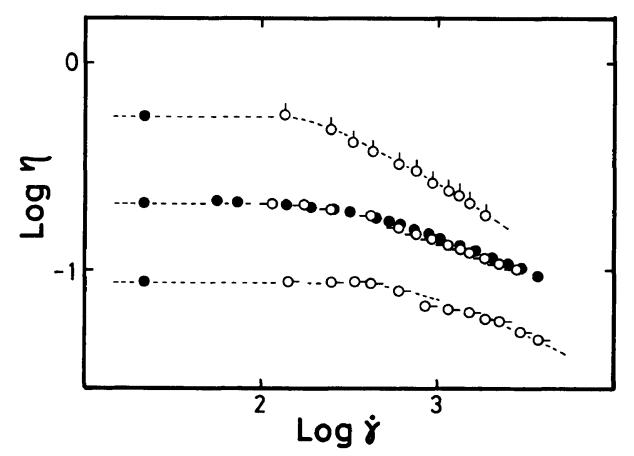

Figure 5. Shear rate dependence of solution viscosity $\eta$. Sample: F-288. The concentrations are $0.566\left(\mathrm{O}^{-}\right)$, $0.962(-\bigcirc)$, and $1.41(\bigcirc) \mathrm{g} \mathrm{dl}^{-1}$. The symbol $\bigcirc$ denotes the data from MKS viscometer.

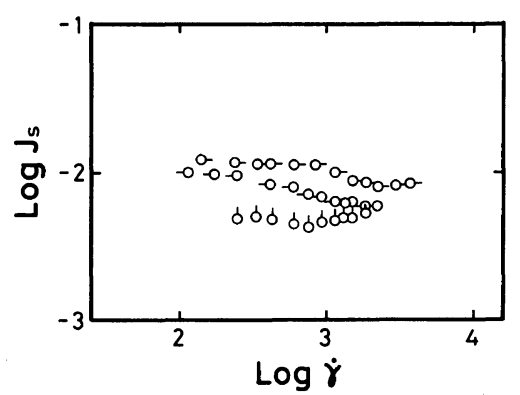

Figure 6. Shear rate dependence of the apparent steady-state compliance $J_{\mathrm{s}}$. The symbols are the same as in Figure 5. 
$\mathrm{dyn}^{-1}$, which was reported by JaneschitzKriegl. ${ }^{1}$ As shown in Figure $4, P_{12}$ and $P_{11}-$ $P_{22}$ are proportional to $\dot{\gamma}$ and $\dot{\gamma}^{2}$, respectively, at low shear rates, but deviate from these proportionalities at higher shear rates.

Figure 5 shows some examples of the shear rate dependence of viscosity at various concentrations evaluated from $P_{12}$, together with the viscosity data of the same sample solutions measured by a Maron-Krieger-Sisko (MKS) capillary viscometer. ${ }^{9}$ As shown in the figure, both viscosity data from the flow birefringence apparatus and the MKS viscometer are in good agreement with each other not only in the Newtonian but also in the non-Newtonian region. Therefore, it may be concluded that the experimental data measured by the present apparatus is reliable and the stress-optical coefficient of Janeschitz-Kriegl can be used over the entire range of shear rates employed in this experiment.

Figure 6 shows the shear rate dependence of the apparent steady-state compliance $J_{\mathrm{s}}$ of the

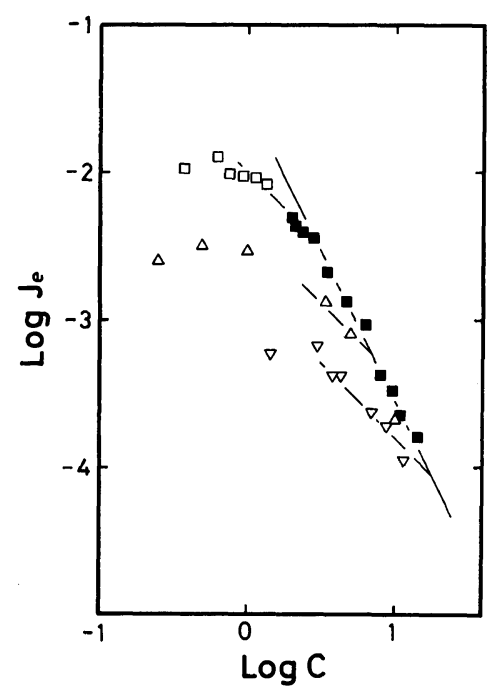

Figure 7. Concentration dependence of the steadystate compliance $J_{\mathrm{e}}$. The symbols $\square$ and $\mathbf{\square}$ denote the data obtained from the flow birefringence apparatus and the Weissenberg rheogoniometer, respectively. Sample: F-450. The symbols $\triangle$ and $\nabla$ denote the data of Daum et al. $\left(\bar{M}_{w}=1.8 \times 10^{6}\right)$ and Ferry et al. $\left(\bar{M}_{w}=8.6 \times 10^{5}\right)$, respectively. same sample solutions as in Figure 5. In Figure 7 the steady-state compliance $J_{\mathrm{e}}$, which is the value at the limit of zero shear rate, is double logarithmically plotted against concentration, together with the data obtained by a Weissenberg rheogoniometer for the same sample (F-450). ${ }^{8}$ The data of polystyrenes with narrow molecular weight distributions as reported by Daum et al. ${ }^{10}$ and Ferry et al. ${ }^{11}$ are also shown in the figure. Although the concentration range available for flow birefringence measurements is different from that for the Weissenberg rheogoniometer, the data from the flow birefringence and from the Weissenberg rheogoniometer are found to compose a uniform line. It is already known that $J_{\mathrm{e}}$ is proportional to $c^{-2}$ at high polymer concentrations (in the entangled region) and to $M / c$ in more dilute solutions (in the Rouse region) ${ }^{8}$ In Figure $7, J_{\mathrm{e}}$ is found to be proportional to $c^{-1}$ in agreement with the previous conclusion if the polymer concentrations are higher than $c a .1 \mathrm{~g} \mathrm{dl}^{-1}$. At the lower concentrations, however, the deviation from the line is clear. The same tendency was already pointed out in the data of Daum et al. ${ }^{10}$ and Ferry et al. ${ }^{11}$

\section{DISCUSSION}

The viscosity data may be normalized as $\left(\eta-\eta_{\mathrm{s}}\right) /\left(\eta^{0}-\eta_{\mathrm{s}}\right)$ where $\eta^{0}$ is the viscosity at the limit of zero shear rate. The shear rate dependence of the normalized values at various concentrations are superposed by shifting the data horizontally as shown in Figure 8. The shift factor $a_{\mathrm{c}}$ assumed for superposition is plotted against polymer concentration in comparison with $\left(\eta^{0}-\eta_{\mathrm{s}}\right) J_{\mathrm{e}}$, which is proportional to the longest relaxation time, in Figure 9. Both are found to be parallel. It can be observed that the master curves thus obtained behave differently in non-entangled and entangled regions. The transition may occur at $c / c^{*}=4 \sim 5$, in agreement with the beginning of cross-over region from the dilute solution to 


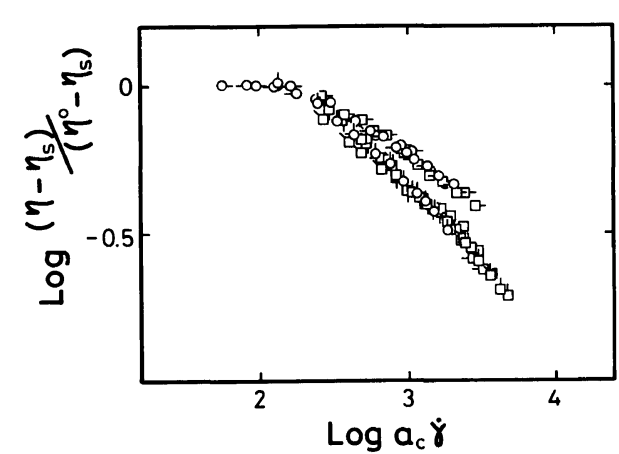

Figure 8. Superposition of shear rate dependence data of reduced viscosity $\left(\left(\eta-\eta_{\mathrm{s}}\right) /\left(\eta^{0}-\eta_{\mathrm{s}}\right)\right)$. The symbols 0 and $\square$ denote the data of F-288 and F-450, respectively. The concentrations are $0.566\left(\bigcirc^{-}\right), 0.962(-\bigcirc), 1.41$ (○), 0.372 ( $\square-), 0.756(\square), 0.936(-\square), 1.12$ ( $\square)$, and 1.34 ( $\square) \mathrm{g} \mathrm{dl}^{-1}$.

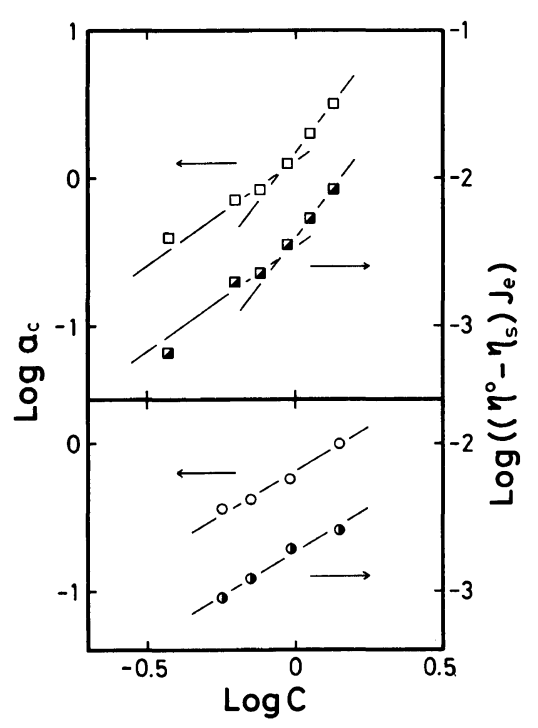

Figure 9. Concentration dependences of shift factor $a_{\mathrm{c}}$ and the relaxation time $\left(\eta_{0}-\eta_{\mathrm{s}}\right) J_{\mathrm{e}}$. The symbols $\bigcirc$ and $\square$ are the same as in Figure 8. The symbols and $\square$ denote the relaxation times of F-288 and F-450, respectively.

the semidilute solution in the piot of $\eta_{\mathrm{sp}} / c[\eta]$ vs. $c / c^{*} \cdot{ }^{7,8}$

Figure 10 shows plots of $J_{\mathrm{s}} / J_{\mathrm{e}}$ against $a_{\mathrm{c}} \dot{\gamma}$ at various concentrations. In contrast to the behavior of viscosity, $J_{\mathrm{s}} / J_{\mathrm{e}}$ is not sensitive to $\dot{\gamma}$ and polymer concentration, as was previously reported for more concentrated solu-

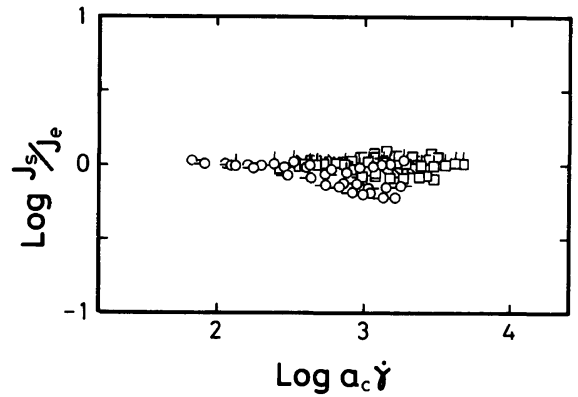

Figure 10. Superposition of shear rate dependence data of reduced compliance $\left(J_{\mathrm{s}} / J_{\mathrm{e}}\right)$. The symbols are the same as in Figure 8.

tions. $^{12}$

The viscoelastic properties of dilute polymer solutions at the infinite dilution and the limit of zero shear rate were well analysed by Rouse $^{13}$ and Zimm, ${ }^{14}$

$$
\begin{gathered}
P_{12}=\left[\eta_{\mathrm{s}}+(c R T / M) \cdot \Sigma \tau_{i}\right] \dot{\gamma} \\
P_{11}-P_{22}=2(c R T / M) \cdot \Sigma \tau_{i}^{2} \cdot \dot{\gamma}^{2}
\end{gathered}
$$

where $\tau_{i}$ is the relaxation time associated with the $i$-th internal mode, $R$ is the gas constant and $T$ is the absolute temperature. From eq $1 \mathrm{a}$, $1 \mathrm{~b}, 7 \mathrm{a}$, and $7 \mathrm{~b}$, we have the reduced steadystate compliance $J_{\mathrm{eR}}$,

$$
\begin{gathered}
J_{\mathrm{e}} \cdot(c R T / m) \cdot\left[\eta^{0} /\left(\eta^{0}-\eta_{\mathrm{s}}\right)\right]^{2}=J_{\mathrm{eR}} \\
=\Sigma \tau_{i}^{2} /\left(\Sigma \tau_{i}\right)^{2}
\end{gathered}
$$

In $\theta$-solvents we have $J_{\mathrm{eR}}=0.206^{14}$ if there is a strong hydrodynamic interaction so that the polymer coil behaves like a non-draining sphere as actually observed for the intrinsic viscosity, while we have $J_{\mathrm{eR}}=0.4^{13}$ if we assume that there is no hydrodynamic interaction between segments so that the polymer coil behaves like a free-draining sphere. In good solvents, on the other hand, $J_{\mathrm{eR}}$ has an intermediate value between them due to excluded volume effects. ${ }^{15}$ As the concentration increases, moreover, $J_{\mathrm{eR}}$ is reported to increase from the value at the infinite dilution and approach to the value for the freedraining sphere because segments of other chains penetrating into a polymer domain 


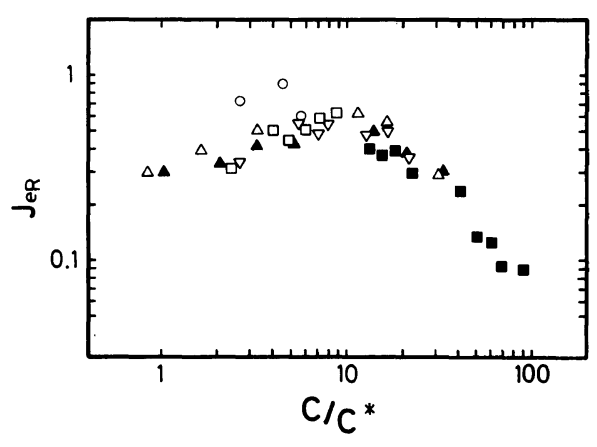

Figure 11. Dependence of the reduced steady-state compliance $J_{\mathrm{eR}}$ on the degree of coil-overlapping $c / c^{*}$. The symbols $\bigcirc$ and $\square$ are the same as in Figure 8. The symbols $\square, \triangle$, and $\nabla$ are the same as in Figure 7. The symbol $\Delta$ denotes the data of Daum et al. ${ }^{11} \quad\left(\bar{M}_{w}=\right.$ $\left.4.1 \times 10^{5}\right)$.

screens the intramolecular hydrodynamic interactions. ${ }^{15}$

Since the interpenetration of polymer chains may be a function of the degree of coiloverlapping, $J_{\mathrm{eR}}$ calculated from the present data is plotted against $c / c^{*}$, together with the data of Daum et al. ${ }^{10}$ and Ferry et al. ${ }^{11}$ in Figure 11. Although the data of F-288 are somewhat higher than other data, the deviation is almost the same magnitude as reported in a literature. ${ }^{16}$ Thus, It may be concluded that $J_{\mathrm{eR}}$ has a plateau or, strictly speaking, a rather broad maximum and decreases to approach to the limiting values for the nondraining coil with decreasing $c / c^{*}$ in the dilute region for $J_{\mathrm{e}}$. It is interesting that $J_{\mathrm{eR}}$ starts to decrease at $c / c^{*}=4-5$ corresponding to the concentration where the non-Newtonian behavior in viscosity changes as described above. Moreover, since $\left(\eta^{0}-\eta_{\mathrm{s}}\right) / \eta_{\mathrm{s}}$ as well as $J_{\text {eR }}$ decrease with decreasing concentration, it is natural that $J_{\mathrm{eR}}$ should show a deviation from the line in the Rouse region as observed in Figure 7.

\section{REFERENCES}

1. H. Janeschitz-Kriegl, Adv. Polym. Sci., 6, 170 (1969).

2. V. N. Tsvetkov, "Newer Methods of Polymer Characterization," B. Ke, Ed., Interscience, New York, N. Y., 1964, p 563.

3. K. Matsuura, S. Hattori, I. Noda, and $\mathbf{M}$. Nagasawa, Optics and Laser Technology, 10, 237 (1978).

4. Y. Higo, N. Ueno, and I. Noda, Polym. J., 15, 367 (1983).

5. I. Noda, Y. Higo, N. Ueno, and T. Fujimoto, Macromolecules, 17, 1055 (1984).

6. I. Noda, N. Kato, T. Kitano, and M. Nagasawa, Macromolecules, 14, 668 (1981).

7. Y. Takahashi, Y. Isono, I. Noda, and M. Nagasawa, Macromolecules, 18, 1002 (1985).

8. Y. Takahashi, I. Noda, and M. Nagasawa, Macromolecules, 18, 2220 (1985).

9. S. H. Maron, I. M. Krieger, and A. W. Sisko, J. Appl. Phys., 25, 971 (1954).

10. U. Daum and J. L: S. Wales, Polym. Lett., 7, 459 (1969).

11. L. A. Holmes and J. D. Ferry, J. Polym. Sci., C, 23, 291 (1968).

12. H. Endo, T. Fujimoto, and M. Nagasawa, J. Polym. Sci., A-2, 9, 345 (1971).

13. P. E. Rouse, J. Chem. Phys., 21, 1272 (1953).

14. B. H. Zimm, J. Chem. Phys., 24, 269 (1956).

15. J. D. Ferry, "Viscoelastic Properties of Polymers," John Wiley \& Sons, Inc., New York, N. Y., 1980, Chapter 9.

16. W. W. Graessley, Adv. Polym. Sci., 16, 66 (1974). 